\title{
Innovative First Step toward Mechanoluminescent Ubiquitous Light Source for Trillion Sensors
}

\author{
Nao Terasaki* \\ Measurement Solution Research Center, \\ National Institute of Advanced Industrial Science and Technology (AIST), \\ 807-1 Shuku-machi, Tosu, Saga 841-0052, Japan \\ (Received February 2, 2016; accepted May 16, 2016)
}

Keywords: mechanoluminescence, elasticoluminescent material, ubiquitous light source

Recently, there have been innovative mechanoluminescent (ML) particles made available, each of which repeatedly emits light in response to small applied stresses even in an elastic region. When dispersedly coated onto a structure, each particle acts as a sensitive mechanical sensor, while the two-dimensional emission pattern of the whole assembly reflects the dynamical stress distribution inside the structure and the mechanical information around cracks and defects. On the other hand, ML ubiquitous light sources, the other final goal of mechanoluminescence, have been discussed and investigated toward innovative nano-light sources, which can be utilized anytime and anywhere, even in deep tissue and cells for an energy harvesting system for trillion sensors. In this review, we would like to focus on the activity toward the ML ubiquitous light sources, and introduce them from the next four viewpoints; the potential of the ML light source, the performance of a single ML ubiquitous light source, nondestructive and noninvasive mechanical stimulation, and application.

\section{Introduction}

Elasticoluminescent material is a novel mechanoluminescent (ML) functional ceramic powder [controllable size: $10 \mathrm{~nm}-100 \mu \mathrm{m}$, representative and most efficient ML material: europium-doped strontium aluminate (denoted as $\mathrm{SrAl}_{2} \mathrm{O}_{4}: \mathrm{Eu}^{2+}$, or SAOE)] that can emit intensive light repeatedly accompanied by mechanical stress such as deformation [Fig. 1(a)], friction, and impact, even in an elastic deformation region. ${ }^{(1-4)}$ The ML intensity is proportional to the strain energy of the material. Thus, when dispersedly coated onto a structure, each particle acts as a sensitive mechanical sensor, while the two-dimensional (2D) emission pattern of the whole assembly reflects the dynamical strain/stress distribution [Fig. 1(b)-(i)] and the mechanical information around defects and cracks, even exciting inside the structure, at the back [Fig. 1(b)-(ii)], or at an invisible crack tip [Fig. 1(b)-(iii)].(5,6) Furthermore, the validity of the information was clearly supported by a simulation of stress distribution [Fig. 1(b)-(i)]. This ML visualization technique has provided a novel way of diagnosing structural health. Actually, the ML sensor has been applied to real infrastructure such as bridges, ${ }^{(7-9)}$ buildings, ${ }^{(10)}$ welding points of pipelines, ${ }^{(6)}$ and high-pressure vessels, ${ }^{(6,11)}$ and

*Corresponding author: e-mail: nao-terasaki@aist.go.jp 


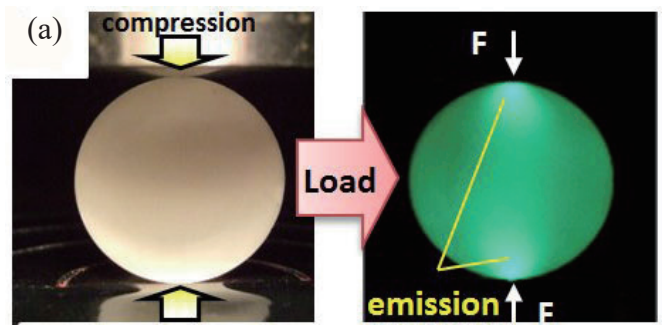

(b)

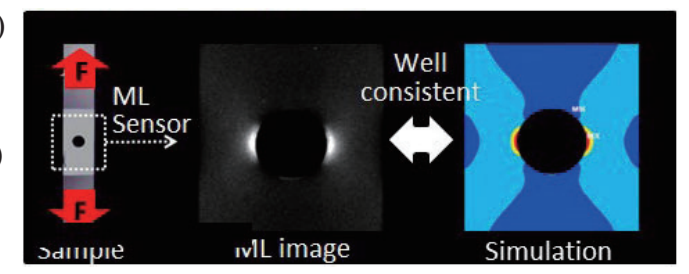

(ii)

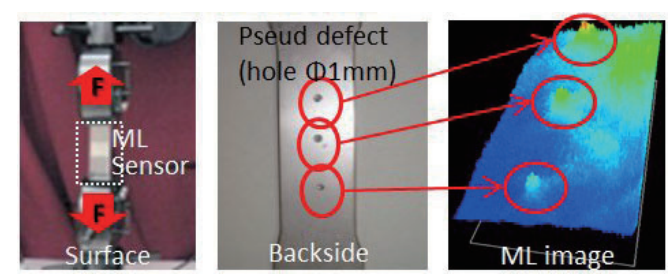

(iii)

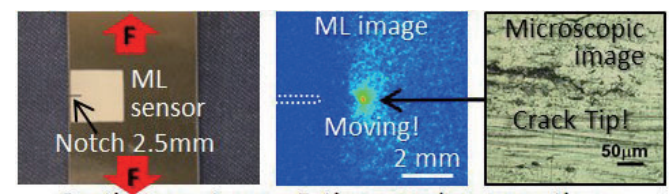

Continuous stress $=$ Fatigue crack propagation

Fig. 1. (Color online) (a) Mechanoluminescence from pellet consisting of ML particle and epoxy resin. Load: compressional load, up to $1000 \mathrm{~N}$. (b) Feature of ML sensor; visualization of (i) 2D/3D stress distribution, (ii) invisible defect at backside, and (iii) tip of microcrack and its propagation.

successfully demonstrated the ability to detect active cracks including information on the likelihood for growth, real crack propagation, and mechanically weak points. At present, many other researchers have also started to develop novel ML materials, sensors, and diagnostic methods. ${ }^{(12-14)}$

Meanwhile, the Trillion Sensors Universe, in which over trillions of sensors cover an entire society, has been starting. The number of sensors in the universe will be 100 times the present requirement with 150 new sensors per year for each of the 7 billion people worldwide. The tremendous number of sensors has been promising to accelerate information oriented appropriate decision, to achieve society without unconscious loss, and to bring abundance and solution on worldwide problems, such as personal health, agricultural production, environmental monitoring, and green energy production. ${ }^{(15)}$ For sensors in the Trillion Sensors Universe, the next properties are promising; 1) ubiquitousness to obtain information in an appropriate time and place and 2) energy harvesting function for self-contained drive.

In the above sense, ML micro- and nanoparticles have great potential as light sources, for example, for solar cells, ${ }^{(16)}$ a historical-log recording system for stress using ML materials and photo-recording materials, ${ }^{(17)}$ and fluorescent molecular probes for bioimaging ${ }^{(18)}$ and phototherapy. ${ }^{(6,19,20)}$ Furthermore, the size of ML nanoparticles, several tens of nanometers, is sufficiently small for injection against bio-body. ${ }^{(21,22)}$ It can be expected that ML particles will act as ubiquitous light sources or ubiquitous energy harvesting systems for the Trillion Sensors Universe, which can be used anytime and anywhere even in the human body and deliver photons to photofunctional materials and trillion sensors existing inside the body for phototherapy, diagnosis, and imaging. ${ }^{(23-25)}$ 
Here, we review the recent innovative activities through the following four aspects and discuss the potential to achieve one of the final goals of ML materials as ML ubiquitous light sources (Fig. 2).

(1) Materials and potential to achieve an ML light source

(2) Performance of single ML particles as ubiquitous light sources

(3) Nondestructive and noninvasive mechanical stimulation for ML generation

(4) Potential applications of ML ubiquitous light sources

\section{ML Materials as Light Sources}

As described in Introduction, SAOE has been frequently used for structural health monitoring and stress/strain visualization, because SAOE microparticles (ca. $1 \mu \mathrm{m}$, Fig. 3) are one of the most efficient ML materials. ${ }^{(5,6)}$ The emission peak is located at a wavelength of $520 \mathrm{~nm}$ (photon energy at $520 \mathrm{~nm}$ can be calculated as $3.82 \times 10^{-19} \mathrm{~W} /$ photon) and is produced by the transition of $\mathrm{Eu}^{2+}$ ions between $4 \mathrm{f}^{7}$ and $4 \mathrm{f}^{6} 5 \mathrm{~d}^{1}$ in SAOE. In addition, the size can be controlled from ca. $10 \mathrm{~nm}-100 \mu \mathrm{m}$ through various synthetic methods, such as the solid phase, microspray, and the use of micelles. ${ }^{(6,26)}$ Sizes smaller than $100 \mathrm{~nm}$ are to be appropriate for injection into a living body, and the size shown here clears the qualification for the final goal.

ML materials with a shorter wavelength consisting of higher energy photons must be more appropriate for use as a light source. For example, $\mathrm{TiO}_{2}$ photocatalysts efficiently absorb ultraviolet (UV) light for activation, ${ }^{(25)}$ and photo-responsible materials for photo-recording and switching also efficiently respond to blue and green light.(23,24) For appropriate combinations of ML light sources and photosensitive materials, we have succeeded in developing ML materials with various emission colors, ${ }^{(1-4,6,27-35)}$ especially with UV, ${ }^{(27)}$ blue, ${ }^{(27-33)}$ and green ${ }^{(1-4,34)}$ achieved so far, as shown in Fig. 4. Although some of the ML materials show emissions enough strong to be seen with the naked eye, the development of ML materials with stronger emissions has remained a challenge to providing efficient light sources.

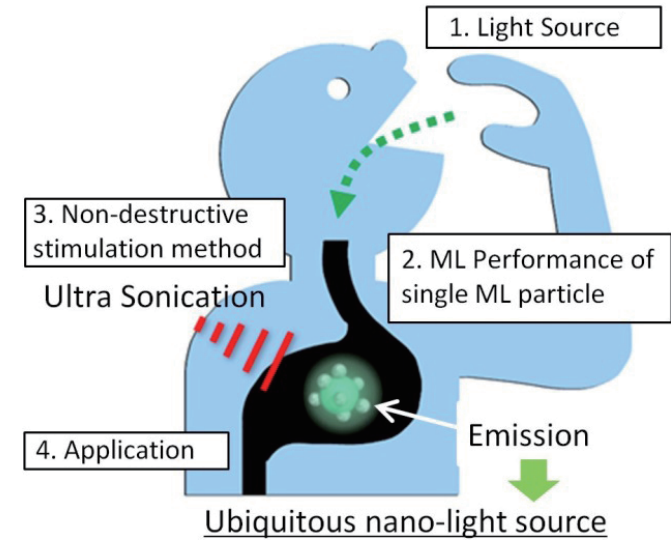

Fig. 2. (Color online) Concept of ubiquitous ML nano-light source and its subjects to be clarified.
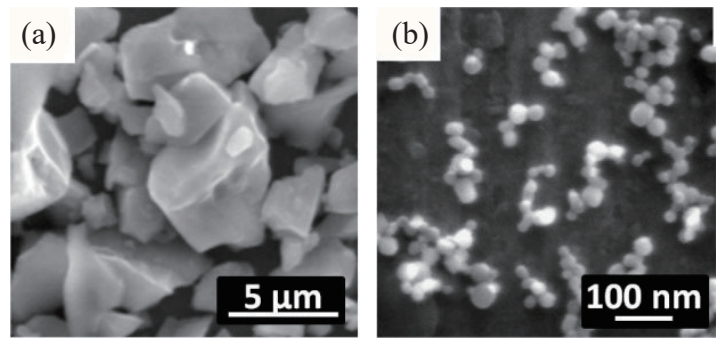

Fig. 3. SEM images of SAOE microparticles synthesized by (a) solid phase method and (b) nanoparticles synthesized by microspray method. 


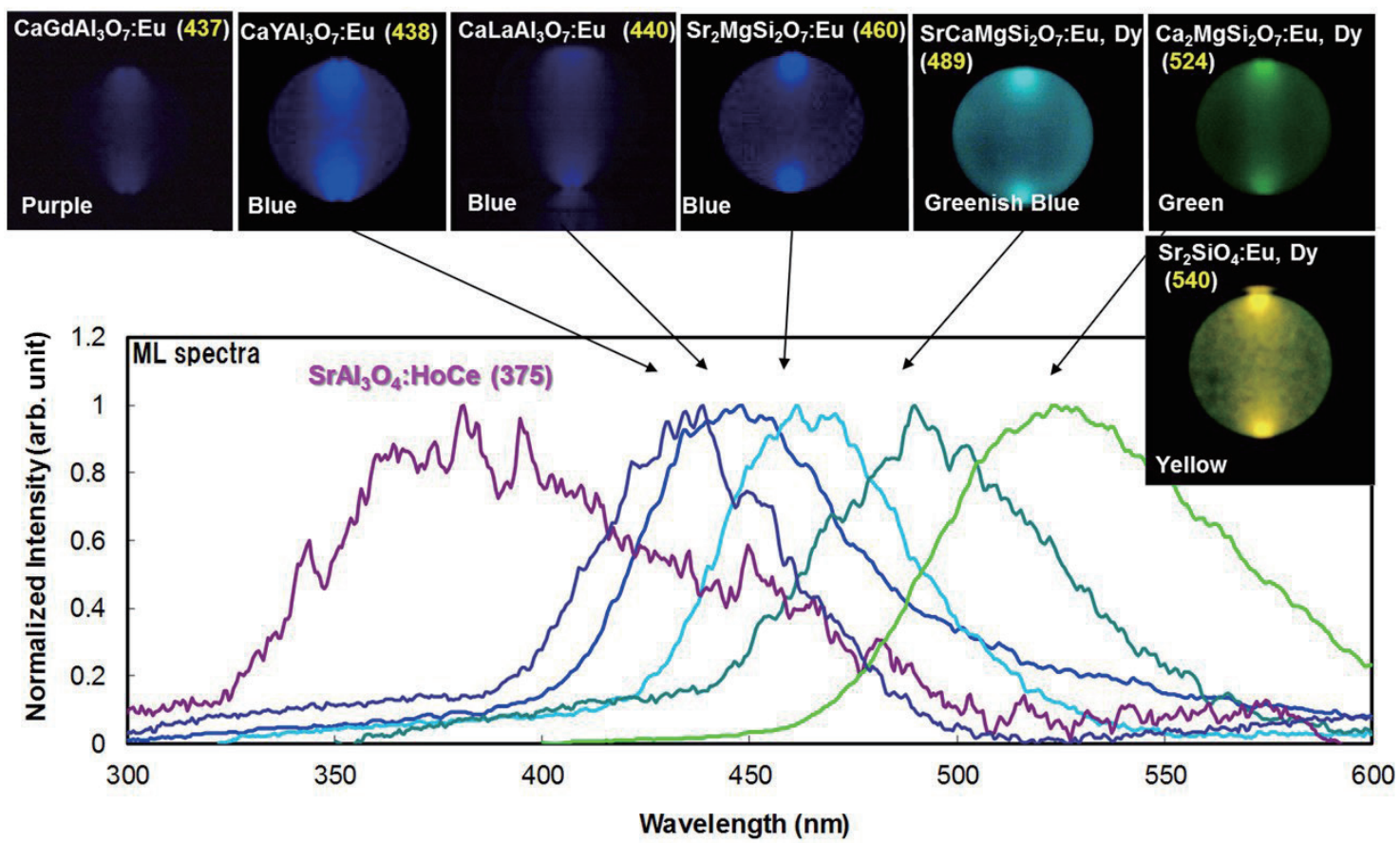

Fig. 4. (Color online) Photographs and ML spectra of UV and blue-emissive ML materials developed for light sources. The numbers in parentheses show the ML wavelength of each ML material.

\section{Potential of ML Light Source}

Mechanoluminescence is an emission phenomenon that arises during stress application. However, emission alone is insufficient for light sources, which should give sufficient light energy to activate other objects. Here, to show the potential of mechanoluminescence as a light source, we introduce examples of ML light sources for photocells and fluorescent probes for bioimaging.

\subsection{ML light source for photocells ${ }^{(16)}$}

As the first step to demonstrate the potential of mechanoluminescence as a light source, a mechanoluminescence-driven photocell system consisting of an ML material and a photocell was prepared. In this system, the ML pellet consisting of SAOE microparticles $\left(\lambda_{\mathrm{ML}}: 520 \mathrm{~nm}\right)$ and epoxy resin was used as a light source, and a silicon solar cell $\left(\lambda_{\text {abs }}>300 \mathrm{~nm}\right)$ was used as a photoelectric converter. As shown in Fig. 5(a), the ML pellet and solar cell were set at perpendicular positions, and a compressional load was applied to the ML pellet using a universal testing machine to generate mechanoluminescence. As a result, photocurrent was successfully observed with the same response curve as the mechanoluminescence, as shown in Fig. 5(b), demonstrating that mechanoluminescence could drive other photofunctional devices and materials, in this case a solar cell, as a light source. 


\subsection{ML light source for fluorescent probes for bioimaging(18)}

Bioimaging has emerged as a promising technology for unraveling the mysteries of life. ${ }^{(23,24)}$ However, the development of an appropriate excitation source for fluorescent probes used in in vivo bioimaging is a challenging task. Thus, we have investigated the excitation of fluorescein isothiocyanate isomer-I (FITC) by the mechanoluminescence emission from $\mathrm{Eu}^{2+}$-doped $\mathrm{CaYAl}_{3} \mathrm{O}_{7}$ (CYAE). ${ }^{(18)}$ CYAE shows intense blue photoluminescence (PL) and mechanoluminescence at approximately $440 \mathrm{~nm}$ with an intensity of $10 \mathrm{nW} / \mathrm{cm}^{2}$ for a load of $1000 \mathrm{~N}$ and a loading speed of $3 \mathrm{~mm} / \mathrm{min}$; this peak is attributed to the transition of $\mathrm{Eu}^{2+}$ between the ${ }^{8} \mathrm{~S}_{7 / 2}\left(4 \mathrm{f}^{7}\right)$ ground state and the $4 \mathrm{f}^{6} 5 \mathrm{~d}^{1}$ excited state. ${ }^{(8,35-37)}$ FITC dye, whose structural formula is shown in Fig. $6(\mathrm{a})$, is one

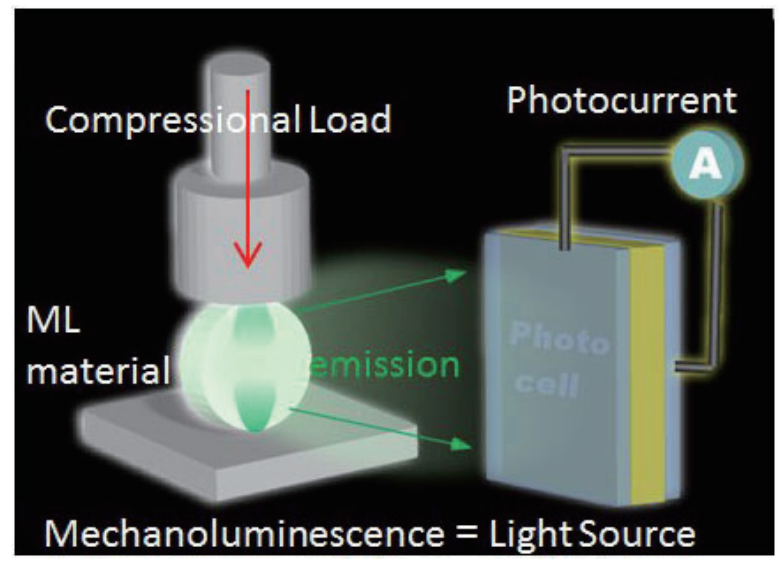

(a)

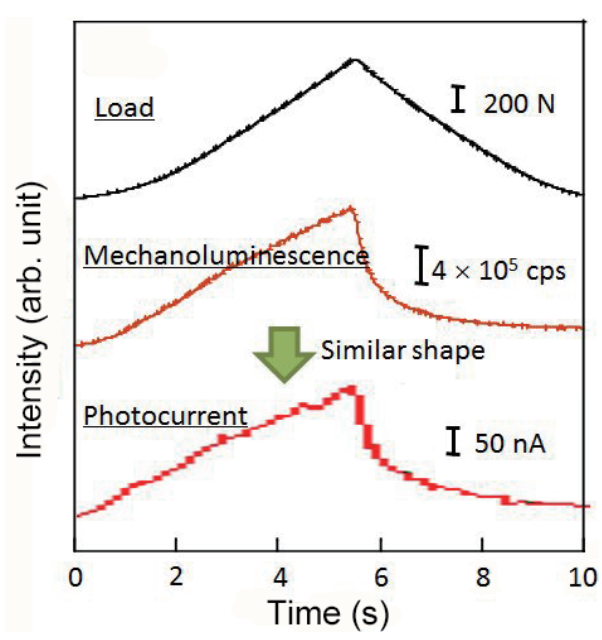

(b)

Fig. 5. (Color online) (a) Illustration of ML light source for photocell. (b) ML response of ML pellet and photocurrent from photocell accompanied by compressional load application up to $1000 \mathrm{~N}$.

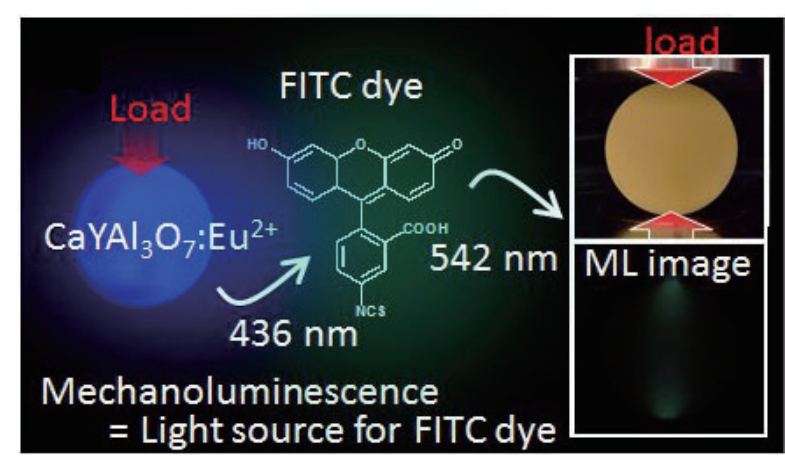

(a)

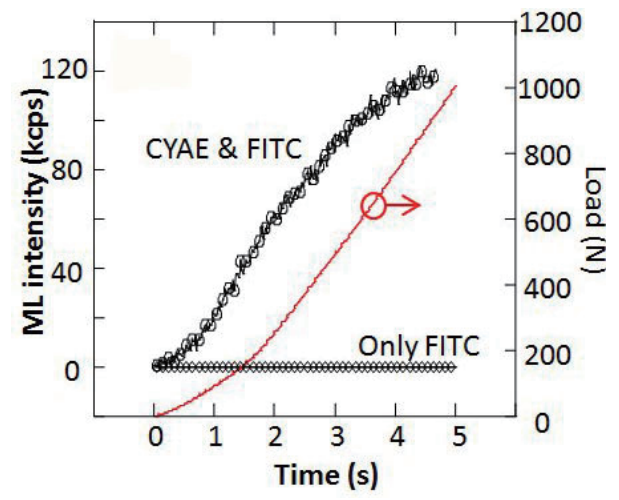

(b)

Fig. 6. (Color online) (a) Illustration of ML light source used for FITC bioimaging probe. (b) ML response of ML pellet including both CYAE and FITC and that including only FITC. Condition: compressional load application up to $1000 \mathrm{~N}$. 
of the most widely used fluorescent markers for labeling amino acids, peptides, enzymes, sugars, viruses, and cells in bioimaging and biodiagnosis. ${ }^{(38)}$ As a result of compressional load application to a pellet composed of both CYAE and FITC [O: Fig. 6(b)], green mechanoluminescence [wavelength: $536 \mathrm{~nm}$; Fig. 6(a)] increased proportionally to an increase in the compressive load (solid line) despite the fact that this dye does not exhibit mechanoluminescence [ $\diamond$ : Fig. 6(b)]. To the best of our knowledge, this study is the first to demonstrate that ML materials can be used as in situ light sources for fluorescent probe molecules.

\section{Performance of Single ML Particles as Ubiquitous Light Sources ${ }^{(39)}$}

In the above cases, although ML particles certainly acted as ubiquitous light sources, a crucial question still remains; how intense is the mechanoluminescence emitted from a single ML particle, in other words, how good is ites performance as a ubiquitous light source? This is closely related to the kinds of possible and suitable future applications; thus, the quantitative evaluation of the performance of a single ML particle is indispensable for use of the ML particle as a ubiquitous ML light source. Previously, we investigated mechanoluminescence from single ML microparticles using an atomic force microscope (AFM) and a photomultiplier. ${ }^{(40)}$ However, here, to make more quantitative discussion, we introduce the evaluation of mechanoluminescence using an ML film with a low ratio of ML particles $(1.0 \mathrm{wt} \%)$ and a photomultiplier to focus on a single ML particle. ML response curves accompanied by tensional loads of 2.5 through $8 \mathrm{kN}$ were measured and a representative one at $8 \mathrm{kN}$ is shown in Fig. 7(a). At the timing of $0 \mathrm{~s}$ along the $X$ axis and $30 \mathrm{~s}$ after UV light irradiation, a load was applied with a frequency of $1 \mathrm{~Hz}$. As a result, the response to the load signal was observed in all cases except the case of $2.5 \mathrm{kN}$. The photon numbers in the mechanoluminescence were estimated from the areas of the ML peaks at $1 \mathrm{~Hz}$ and summarized with the corresponding strain value for each ML measurement [Fig. 7(b)]. This indicates that, even for

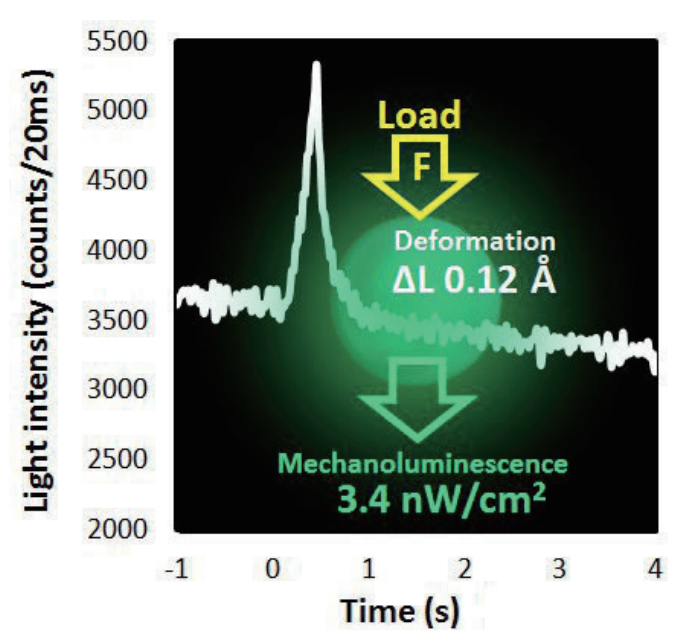

(a)

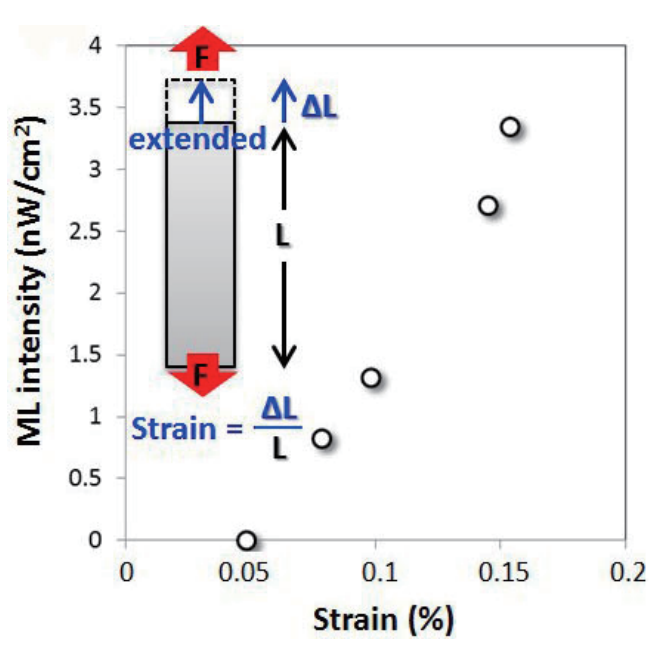

(b)

Fig. 7. (Color online) (a) Performance of single ML particle as ubiquitous light source. (b) ML generating performance of single ML particle, which corresponds to the measured strain value of the ML test piece and calculated deformation value of single ML particle. Load Conditions: tension, 2.5-8 kN. 
the performance of a single ML, higher load and strain give more intense mechanoluminescence. ML performance of a single ML particle was estimated as $3.4 \mathrm{nW} /$ particle at $8 \mathrm{kN}$, by considering that the number of SAOE particles was 288 in the measurement area of the photomultiplier, the area of a single SAOE particle was $1 \mu \mathrm{m}^{2}$, and the energy of a single photon at a wavelength of $520 \mathrm{~nm}$ was $3.82 \times 10^{-19} \mathrm{~W} /$ photon. The SAOE ML particle can act as a nW light source, and the intensity can be controlled via the intensity of the mechanical signal applied.

\section{Nondestructive and Noninvasive Mechanical Stimulation for ML Generation ${ }^{(19)}$}

We have focused on ultrasonic waves as the mechanical stimulation for the generation of mechanoluminescence, because ultrasonic waves have already been utilized for the nondestructive and noninvasive inspection in the construction field and the human body. Furthermore, ultrasonic waves show mechanical factors, for example, sound pressure, acoustic radiation force, rapid bubble expansion and collapse by cavitation, and pressure gradient between nodes and loops in standing waves, among others. ${ }^{(41)}$ For experiments on ultrasonic-wave-induced mechanoluminescence (USML), an SAOE ML particle was blended into an adhesive matrix and immobilized at the bottom of a sample tube at a depth of $2 \mathrm{~mm}$. The sample tube was set at the center of a water bath $\left(300 \times 240 \times 85 \mathrm{~mm}^{3}\right)$ in a commercial ultrasonic cleaner, immersed $2 \mathrm{~mm}$ from the bottom, as shown in Fig. 8(a). As a result, clear emissive responses were observed repeatedly from the ML sample tube accompanied by $37 \mathrm{kHz}$ ultrasonic wave irradiation. In addition, Fig. 8(b) clearly

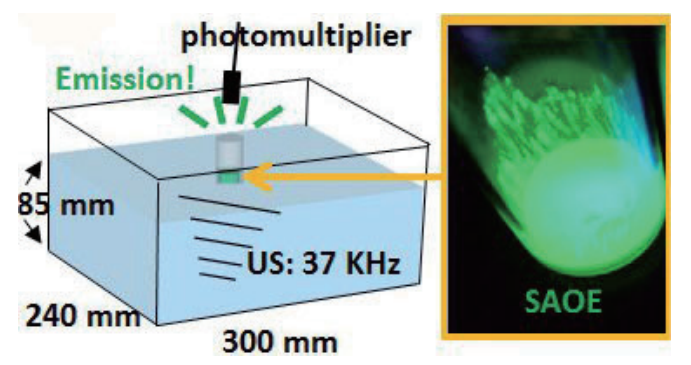

(a)

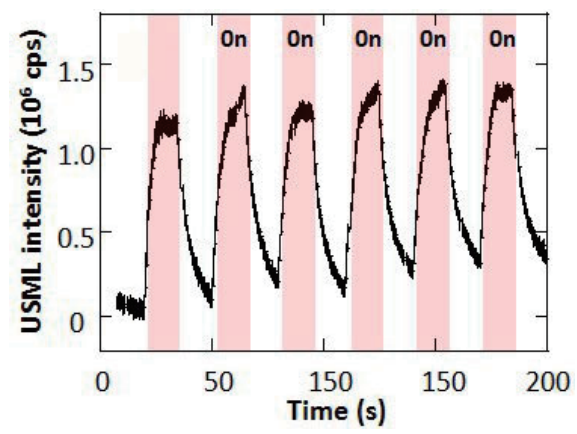

(b)

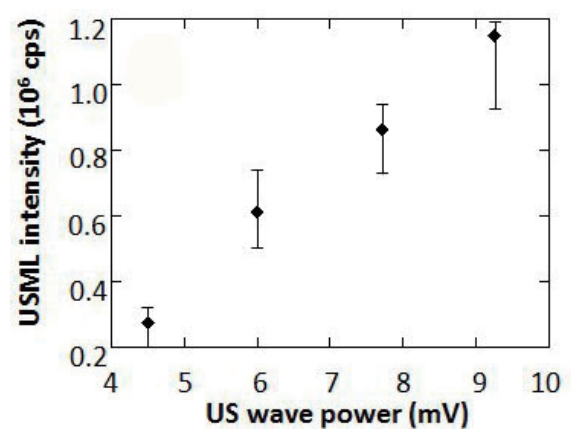

(c)

Fig. 8. (Color online) (a) Experimental setup and photograph of SAOE immobilized in sample tube for USML. (b) Response of emission intensity on ultrasonic irradiation. (c) Correlation between intensity of input ultrasonic wave and USML. Conditions: sample: SAOE, temperature of water bath: $20^{\circ} \mathrm{C}$, frequency: $37 \mathrm{kHz}$. The intensity of the input ultrasonic wave was controlled via the duty ratio. 
shows that the USML intensity from the ML sample tube was proportional to the intensity of the irradiated ultrasonic wave, controlled by changing the duty ratio. In the USML test, downsizing of ML particles was not observed in any condition; namely, the emission was probably not due to the fracture of the ML particle by ultrasonication (fractoluminescence) but to mechanoluminescence. Thus, the same as conventional mechanical stimulation, an ultrasonic wave could be utilized in a smart mechanical stimulation to achieve a ubiquitous light source using ML particles.

\section{Potential Application of ML Ubiquitous Light Source ${ }^{(19,20)}$}

To demonstrate the potential applications of the ubiquitous light source, USML-driven photocatalysis was investigated, as shown in Fig. 9(a). The green mechanoluminescence of SAOE at a wavelength of $520 \mathrm{~nm}$ was expected to act as light energy to activate MPT-623 as visible light responsive $\mathrm{TiO}_{2}$. Therefore, the USML-driven photocatalytic activity tests were carried out using the methylene blue method with Sample 1, including $5 \mathrm{mg}$ SAOE ML particles, and Sample 2, not including ML particles, in $5 \mathrm{ml}$ EtOH solution. The results were summarized with comparison of the results of Samples 1 and 2.

As shown in Fig. 9(b), before ultrasonic wave irradiation (US: 0 h), almost the same and typical absorption spectra of methylene blue dye were detected in both Samples 1 and 2 (US: 0 h). In contrast, only the absorption spectrum in Sample 1 was dramatically changed after $5 \mathrm{~h}$ of ultrasonic wave irradiation (US: $5 \mathrm{~h}$ ). From the summary of the areas of absorption peaks originating from

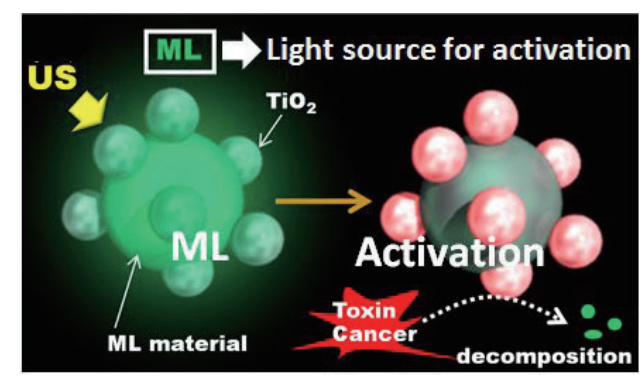

(a)

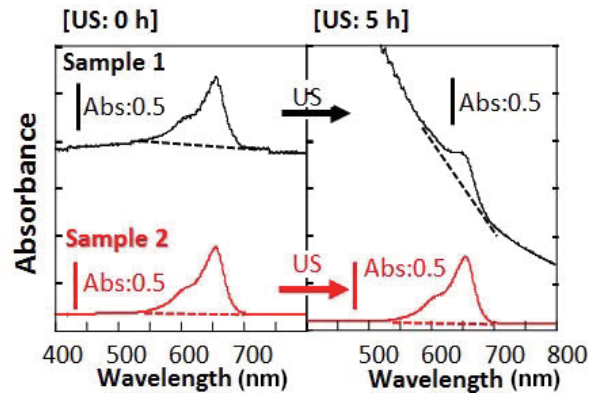

(b)

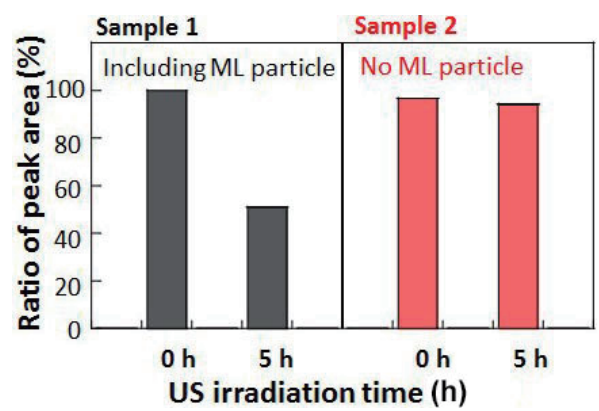

(c)

Fig. 9. (Color online) (a) Illustration of ML ubiquitous light source for activation of $\mathrm{TiO}_{2}$ photocatalysis. (b) Absorption spectra of Sample 1 (including SAOE particles) and Sample 2 (not including SAOE particles) before (US: $0 \mathrm{~h}$ ) and after (US: $5 \mathrm{~h}$ ) ultrasonic wave irradiation. (c) Comparison of areas of absorption peaks originating from methylene blue dye in (b). 
methylene blue dye in Fig. 9(c), almost half of the methylene blue dye was degraded by the ultrasonic wave irradiation only in the case of Sample 1, including ML particles. Moreover, no degradation of the methylene blue dye was detected from the results using Sample 2, not including SAOE ML particles. This difference in the results of methylene blue degradation occurred under the same experimental conditions except for the presence of SAOE ML particles only in Sample 1. It can be concluded that the SAOE ML particles acted as a light source to activate the $\mathrm{TiO}_{2}$ photocatalyst.

\section{Conclusions}

In this review of research moving toward the final goal of mechanoluminescence from ML ubiquitous light sources that can be utilized even in deep tissue and cells, we discussed the potential of the ML light source, the performance of a single ML ubiquitous light source, nondestructive and noninvasive mechanical stimulation, and applications. Currently, further experiments on ML ubiquitous light sources have been ongoing, especially focusing on higher ML intensity, various sizes and shapes of ML particles, evaluation of toxicity, utilization of the surface of ML particles where the highest density field of ML photons, bioimaging, and so on. One of the interesting examples is a UV light emissive ML material, $\mathrm{SrAl}_{2} \mathrm{O}_{4}: \mathrm{HoCe}$, which shows broad emission in the UV wavelength region from 350 to $370 \mathrm{~nm}$. We previously carried out a cell viability test (K562: human erythromyeloblastoid leukemia cell line) using the UV-type ML material. K562 cancer cells died only in the case of the combination of the UV-type ML material and $\mathrm{TiO}_{2}$ photocatalyst. On the other hand, the sterilizing properties were not observed in the case of only each ML material or $\mathrm{TiO}_{2}$ photocatalyst. These results clearly indicate that the ML material does not show toxicity and the UV-type ML material is effective for the activation of the $\mathrm{TiO}_{2}$ photocatalyst. Although this is a preliminary demonstration, we expect the ML material as a ubiquitous light source for phototherapy. The innovative research is just getting started; however, we would like to continue and advance the research to achieve an innovative ubiquitous light source as one of the final goals and killer applications of mechanoluminescence.

\section{Acknowledgements}

This study was partially supported by KAKENHI, Grant-in-Aid for Young Scientists (A), (B) and Exploratory Research, and the Industrial Technology Research Grant Program in 2006 from the New Energy and Industrial Technology Development Organization (NEDO) of Japan. The author thanks the Cooperative Research Project of the Research Institute of Electronics, Shizuoka University. The author also thanks Professor Chao-Nan Xu and all members of the ML group and the trillion sensing group at AIST.

\section{References}

1 C. N. Xu, T. Watanabe, M. Akiyama, and X. G. Zheng: Appl. Phys. Lett. 74 (1999) 2414.

2 C. N. Xu, X. G. Zheng, M. Akiyama, K. Nonaka, and T. Watanabe: Appl. Phys. Lett. 76 (2000) 176.

3 Y. Liu and C. N. Xu: J. Phys. Chem., B 107 (2003) 3991.

4 C. N. Xu: Encyclopedia of Smart Materials 1-2 (2002) 190.

5 C. Li, C. N. Xu, L. Zhang, H. Yamada, and Y. Imai: J. Visualization 11 (2008) 329. 
6 C. N. Xu, N. Ueno, N. Terasaki, and H. Yamada: Mechanoluminescence and Novel Structural Health Diagnosis (Tokyo, NTS, 2012).

7 N. Terasaki and C. N. Xu: Sens. J. IEEE 13 (2013) 3999.

8 N. Terasaki, C. N. Xu, C. Li, L. Zhang, C. Z. Li, D. Ono, M. Tsubai, Y. Adachi, Y. Imai, N. Ueno, and T. Shinokawa: Proc. SPIE SMART STRUCTURES/NDE 2 (2012) 348D1.

9 N. Terasaki, C. N. Xu, C. Li, L. Zhang, Y. Sakata, N. Ueno, K. Yasuda, and L. Ichinose: Proc. 6th Int. Conf. Bridge Maintenance Safety Manage. (2012) 2542.

10 N. Terasaki, C. Li, L. Zhang, and C. N. Xu: 2012 IEEE Sens. Appl. Symp. (SAS 2012) Proc. (2012) p. 77.

11 Y. Fujio, C. N. Xu, Y. Terasawa, Y. Sakata, J. Yamabe, N. Ueno, N. Terasaki, A. Yoshida, S. Watanabe, and Y. Murakami: Int. J. Hydrogen Energy 41 (2016) 1333. doi:10.1016/j.ijhydene.2015.10.073

12 J. S. Kim, K. Kibble, Y. N. Kwon, and K. S. Sohn: Opt. Lett. 34 (2009) 1915.

13 S. M. Jeong, S. Song, S.-K. Lee, and B. Choi: Appl. Phys. Lett. 102 (2013) 051110.

14 S. Someya, K. Ishii, M. Saeki, and T. Munakata: Opt. Lett. 38 (2013) 1095.

15 Roadmap for the Trillion Sensor Universe: http://tsensorssummit.org/Resources/TSensors\%20Roadmap\%20 v1.pdf.

16 N. Terasaki, C. N. Xu, Y. Imai, and H. Yamada: Jpn. J. Appl. Phys. 46 (2007) 2385.

17 N. Terasaki and C. N. Xu: Jpn. J. Appl. Phys. 48 (2009) 04C150.

18 N. Terasaki, H. Yamada, and C. N. Xu: Chem. Commun. 47 (2011) 8034.

19 N. Terasaki, H. Yamada, Y. Imai, and C. N. Xu: Thin Solid Films 518 (2009) 473.

20 N. Terasaki, H. Yamada, and C. N. Xu: Catalysis Today 201 (2013) 203.

21 Q. M. Chermont, C. Chanéac, J. Seguin, F. Pelle, S. Maîtrejean, J. P. Jolivet, D. Gourier, M. Bessodes, and D. Scherman: Proc. Natl. Acad. Sci. U.S.A. 104 (2007) 9266.

22 T. Maldiney, A. Lecointre, B. Viana, A. Bessi, M. Bessodes, D. Gourier, C. Richard, and D. Scherman: J. Am. Chem. Soc. 133 (2011) 11810.

23 P. Alivisatos: Nature Biotech. 22 (2004) 47.

24 G. P. Mitchell, C. A. Mirkin, and L. R. Letsinger: J. Am. Chem. Soc. 121 (1999) 8122.

25 A. Fujishima, J. Ohtsuki, T. Yamashita, and S. Hayakawa: Photomed. Photobiol. 8 (1986) 45.

26 C. Li, Y. Imai, Y. Adachi, H. Yamada, K. Nishikubo, and C. N. Xu: J. Am. Ceram. Soc. 90 (2007) 2273.

27 H. Zhang, H. Yamada, N. Terasaki, and C. N. Xu: Appl. Phys. Lett. 91 (2007) 081905.

28 H. Zhang, H. Yamada, N. Terasaki, and C. N. Xu: J. Electrochem. Soc. 155 (2008) J128.

29 H. Zhang, H. Yamada, N. Terasaki, and C. N. Xu: Electrochem. Solid-State Lett. 10 (2007) J129.

30 H. Zhang, H. Yamada, N. Terasaki, and C. N. Xu: Key Eng. Mater. 88 (2009) 305.

31 H. Zhang, N. Terasaki, H. Yamada, and C. N. Xu: Physica E 8842 (2010) 2872.

32 H. Zhang, N. Terasaki, H. Yamada, and C. N. Xu: Int. J. Modern Phys. B, 23 (2009) 1028.

33 H. Zhang, N. Terasaki, H. Yamada, and C. N. Xu: J. Jpn. Appl. Phys. 48 (2009) 04C109.

34 H. Zhang, N. Terasaki, H. Yamada, and C. N. Xu: Thin Solid Films 518 (2009) 610.

35 H. Zhang, H. Yamada, N. Terasaki, and C. N. Xu: J. Electrochem. Soc. 155 (2008) J55

36 N. Kodama, N. Sasaki, M. Yamaga, and Y. Masui: J. Lumin. 19 (2001) 94.

37 T. Aitasalo, P. Dereń, J. Hölsä, H. Jungner, J. C. Krupae, M. Lastusaari, J. Legendziewicz, J. Niittykoski, and W. Stręk: J. Solid State Chem. 171 (2003) 114.

38 P. V. Chang, J. A. Prescher, M. J. Hangauer, and C. R. Bertozzi: J. Am. Chem. Soc. 129 (2007) 8400.

39 N. Terasaki and C. N. Xu: J. Colloid Interface Sci. 427 (2014) 62.

40 K. Sakai, T. Koga, Y. Imai, S. Maehara, and C. N. Xu: Phys. Chem. Chem. Phys. 8 (2006) 2819.

41 D. Lohse: Nature 434 (2005) 33. 